University of Nebraska - Lincoln

DigitalCommons@University of Nebraska - Lincoln

USDA Forest Service / UNL Faculty Publications U.S. Department of Agriculture: Forest Service -National Agroforestry Center

$12-2008$

\title{
Forest Structure and Downed Woody Debris in Boreal, Temperate, and Tropical Forest Fragments
}

\author{
William A. Gould \\ International Institute of Tropical Forestry GIS and Remote Sensing Laboratory,wgould@fs.fed.us \\ Grizelle Gonzalez \\ USDA Forest Service, International Institute of Tropical Forestry, ggonzalez@fs.fed.us \\ Andrew T. Hudak \\ Rocky Mountain Research Station, ahudak@fs.fed.us \\ Teresa Nettleton-Hollingsworth \\ USDA FS, Boreal Ecology Cooperative Research Unit, thollingsworth@fs.fed.us \\ Jamie Hollingsworth \\ Bonanza Creek Long Term Ecological Research (LTER) program, fsjh@uaf.edu
}

Follow this and additional works at: https://digitalcommons.unl.edu/usdafsfacpub

Gould, William A.; Gonzalez, Grizelle; Hudak, Andrew T.; Nettleton-Hollingsworth, Teresa; and Hollingsworth, Jamie, "Forest Structure and Downed Woody Debris in Boreal, Temperate, and Tropical Forest Fragments" (2008). USDA Forest Service / UNL Faculty Publications. 176.

https://digitalcommons.unl.edu/usdafsfacpub/176

This Article is brought to you for free and open access by the U.S. Department of Agriculture: Forest Service -National Agroforestry Center at DigitalCommons@University of Nebraska - Lincoln. It has been accepted for inclusion in USDA Forest Service / UNL Faculty Publications by an authorized administrator of DigitalCommons@University of Nebraska - Lincoln. 


\section{Forest Structure and Downed Woody Debris in Boreal, Temperate, and Tropical Forest Fragments}

Forest fragmentation affects the heterogeneity of accumulated fuels by increasing the diversity of forest types and by increasing forest edges. This heterogeneity has implications in how we manage fuels, fire, and forests. Understanding the relative importance of fragmentation on woody biomass within a single climatic regime, and along climatic gradients, will improve our ability to manage forest fuels and predict fire behavior. In this study we assessed forest fuel characteristics in stands of differing moisture, i.e., dry and moist forests, structure, i.e., open canopy (typically younger) vs. closed canopy (typically older) stands, and size, i.e., small (10-14 ha), medium (33 to $60 \mathrm{ha}$ ), and large (100-240 ha) along a climatic gradient of boreal, temperate, and tropical forests. We measured duff, litter, fine and coarse woody debris, standing dead, and live biomass in a series of plots along a transect from outside the forest edge to the fragment interior. The goal was to determine how forest structure and fuel characteristics varied along this transect and whether this variation differed with temperature, moisture, structure, and fragment size. We found nonlinear relationships of coarse woody debris, fine woody debris, standing dead and live tree biomass with mean annual median temperature. Biomass for these variables was greatest in temperate sites. Forest floor fuels (duff and litter) had a linear relationship with temperature and biomass was greatest in boreal sites. In a five-way multivariate analysis of variance we found that temperature, moisture, and age/structure had significant effects on forest floor fuels, downed woody debris, and live tree biomass. Fragment size had an effect on forest floor fuels and live tree biomass. Distance from forest edge had significant effects for only a few subgroups sampled. With some exceptions edges were not distinguishable from interiors in terms of fuels.

\section{INTRODUCTION}

North American landscapes are a dynamic matrix of vegetation cover affected by human activity and natural disturbances (1). Human activity includes centuries of agricultural clearing and logging, agricultural abandonment and forest regeneration, and more recently, population expansion and urban sprawl. Fire, drought, disease, insects, and windstorms are large-scale natural disturbances that affect the matrix of vegetation in North America. In this context, fire both controls and responds to patterns of vegetation cover through mutual feedbacks (2). One consequence of natural and human induced dynamics is that the composition and structure of forests, i.e., characteristics such as tree heights and diameters, the quantity and quality of live and dead wood in the forests, the structural and functional characteristics of the forest floor and canopy, and the species composition and biodiversity are spatially variable and difficult to predict across a landscape. Thus, landscape fragmentation creates a complex environment in which to manage forests. Not only do the forest and nonforest components of a landscape add heterogeneity to the matrix, forested fragments themselves can increase heterogeneity due to edge effects $(3,4)$.

Over $40 \%$ of the forests in the continental US are located within $90 \mathrm{~m}$ of a non-forest edge (5). Fragments created by clearcuts and subsequent forest regeneration differ from continuous forest in that they increase the percentage of fragment edges relative to total forest cover and alter the proximity and connectivity of fragments (6). The amount of forest edge is important in two ways: i) edge structure potentially differs from center structure due to differing environmental conditions and ii) edges provide an interface between nonforest and forest conditions that may increase the permeability of abiotic (e.g., light, wind, fire) and biotic (e.g., indigenous and invasive species) components to forest fragment interiors (4). Edge structure may differ from fragment centers in terms of susceptibility to fire (7) and tree mortality (8), and edge area can exceed core area in fragmented forests (6). Edge structure (open vs. closed canopies) significantly affects fragment microclimate (9). The effects of fragmentation on productivity, mortality, and decomposition in forests may vary with fragment size, forest type, climate and the surrounding landscape matrix (3).

\section{Fragmentation and Fuels}

Understanding the interaction between fire, woody biomass, and fragmented landscapes is important for managing wildfire and wildlands because fragmentation and fire are intricately linked. In addition, fragmentation can affect fuel accumulation, increase the spatial variability of fuel loads, and affect the susceptibility of forests to fire. Fuel, or the distribution of carbon in forests, is a key element in determining fire behavior and is the only component of the fire behavior triangle (fuel, topography, weather) that can be effectively managed. Little is known about how fuel loads vary within and between fragments. Although fire potential can be evaluated using climatic and fuel load variables, estimating fuel loads using readily available remote sensing techniques is difficult at best (10). Empirical data on the ecology of fragments suggest that forest composition, structure, and fuel loads are strongly related to the morphology of fragments (area, shape, and perimeter) (9). Remote sensing and field analyses of fragment fuel loads and spatial distributions can lead to better spatial prediction of fuel loads.

Fragmentation can affect fire frequency, increasing the length of the fire cycle in temperate regions (11) and increasing fire susceptibility in tropical regions $(2,12,13)$. This is primarily due to alteration of fuel load patterns. Forest fragmentation can affect the heterogeneity of accumulated fuels in forest wildlands by increasing the diversity of forest types and ages, and by increasing forest edges, which may differ from core areas in composition and structure. This heterogeneity has implications in how we manage fuels, fire, and forests. Understanding the 


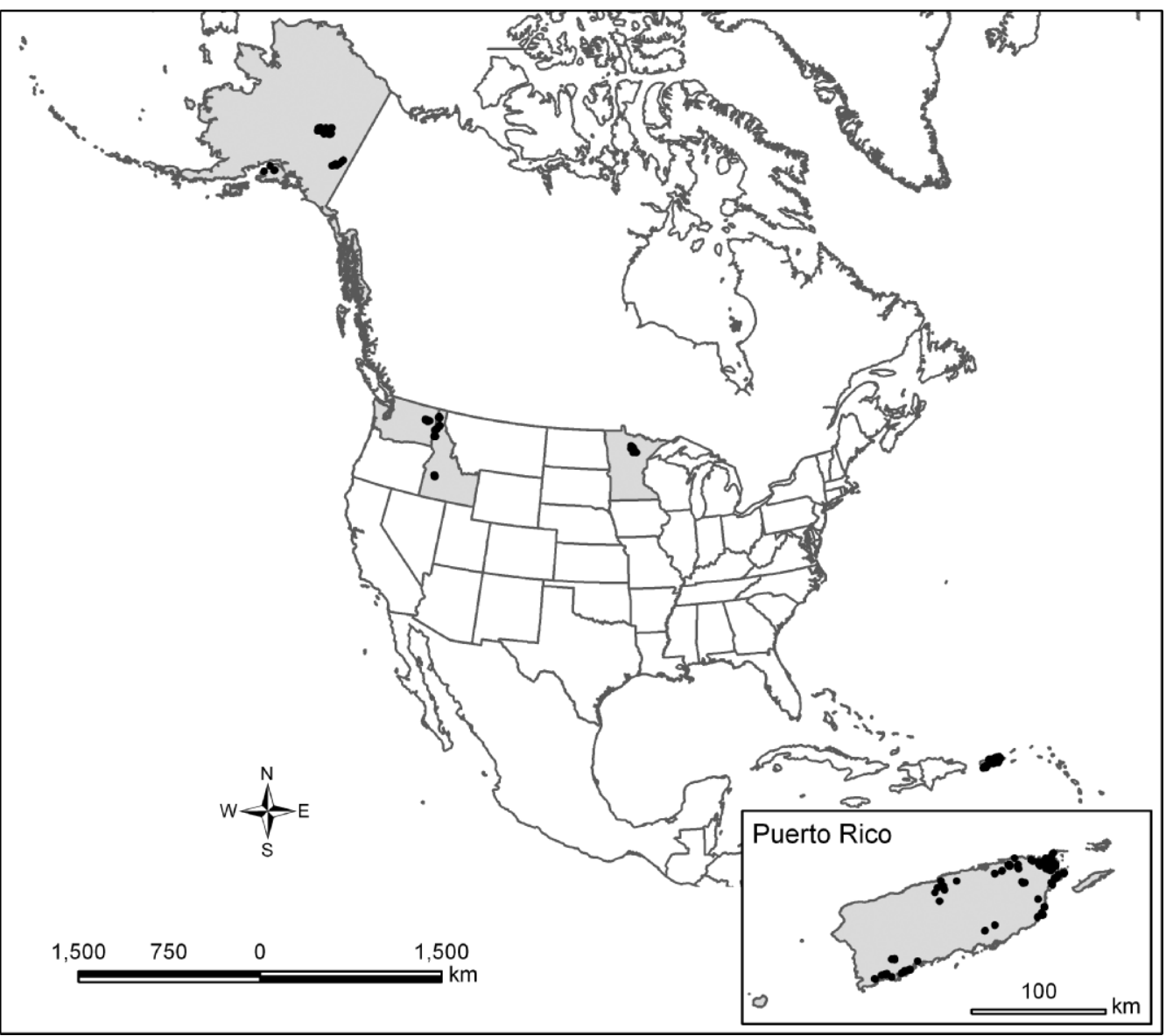

relative importance of fragmentation on variation in fuel loading within a single climatic regime, and the variation in this relationship along climatic gradients, will improve our ability to manage forest fuel load and predict fire behavior in increasingly fragmented forest landscapes. This study represents a comprehensive approach to gaining this understanding in that it analyzes landscapes from tropical to boreal climates, analyzes patterns within fragments and landscapes, and investigates mechanisms controlling fuel load.

In this study we assessed a variety of forest fuel characteristics in forest stands of differing moisture, i.e., dry and moist forests, structure, i.e., open canopy (typically younger) vs. closed canopy (typically older) stands, and size categories, i.e., small (10-14 ha), medium (33 to 60 ha), and large (100-240 ha) along a climatic gradient of boreal, temperate, and tropical climatic zones. In each of these forest types we sampled fragments in a series of plots along a transect running from outside the forest edge to the fragment interior. The goal was to determine how the forest structure and fuel characteristics varied along this transect and whether this variation differed with climate, moisture, structure, and fragment size. Our goal was to examine the relationships between these characteristics and specific fuels attributes, including loadings of fine woody debris (FWD) and coarse woody debris (CWD), standing dead wood, duff and litter layers, canopy height and cover characteristics, and live biomass in order to provide information for modeling fuel loads in fragmented forest landscapes.

\section{METHODS}

\section{Field Sampling}

We established transects crossing from non forest areas, perpendicular to forest edges, and into forest fragment interiors, in a set of moist and dry, open and closed canopy, and small, medium, and large fragments in boreal, temperate, and tropical landscapes in North America (Fig. 1). We sampled evergreen coniferous forests in the boreal and temperate climatic zones, and broadleaf evergreen and semideciduous forests in the tropical climatic zone - see González et al. (14) for detailed description of sites. These forest types are representative of a large part of western and northern North America and the Caribbean. The sites represent a range of managed and unmanaged forests, with the temperate forests being more intensively managed for timber than the boreal or tropical sites. The climatic regions represent a gradient of both temperature and precipitation. Using the PRISM (30 year average) data layers $(15,16)$, the boreal sites mean annual precipitation (MAP) is $407 \mathrm{~mm}$, the temperate sites $922 \mathrm{~mm}$, and the tropical sites $1688 \mathrm{~mm}$. Mean annual median temperatures were $-2^{\circ} \mathrm{C}$ in the boreal sites, $6.3^{\circ} \mathrm{C}$ in the temperate sites, and $25.6^{\circ} \mathrm{C}$ in the tropical sites.

Within each of the three climatic regions we selected representative dry and moist forest types. The dry boreal sites are in central Alaska (MAP $360 \mathrm{~mm}$ ) and the moist boreal sites are in northern Minnesota and the Alaskan Kenai peninsula (MAP $660 \mathrm{~mm}$ ). The dry temperate sites are in eastern Washington State and southern Idaho (MAP $682 \mathrm{~mm}$ ) and the moist temperate sites are in northern Idaho (MAP 1017 $\mathrm{mm})$. The dry tropical sites are in southern and northeastern Puerto Rico along the coast (MAP $1328 \mathrm{~mm}$ ). Most of these plots are within the Holdridge subtropical dry lifezone (17) although some occur in dry-moist transitional sites that are structurally and compositionally similar to subtropical dry forests (18). The moist tropical sites were in the central and northeastern lowlands of Puerto Rico (MAP $1992 \mathrm{~mm}$ ) and within the Holdridge subtropical moist lifezone (17).

Two structural or age classes were selected within each climatic region, an open and a closed canopy forest type. 


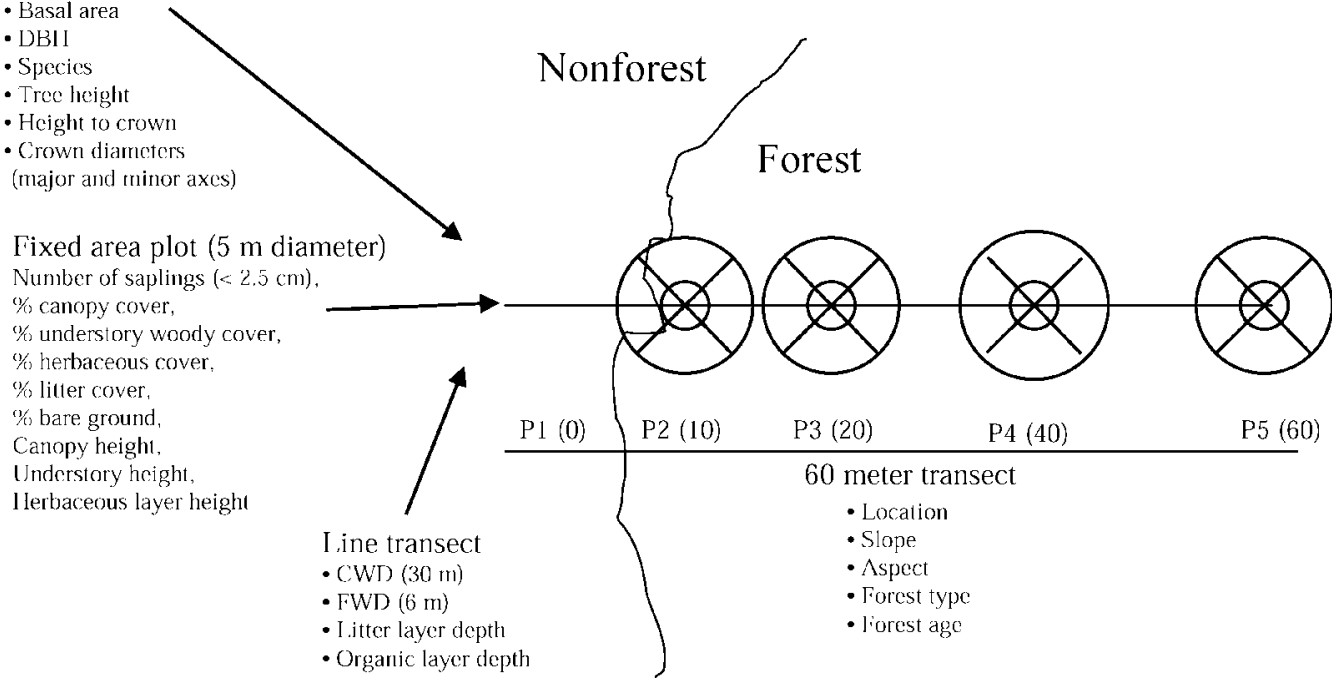

Figure 2. Sampling scheme along transects crossing forest/nonforest boundaries in fragments of various size, climate, and structure/age categories.

Selection was based on field characterization, aerial photography, and ancillary data. The open canopy class has less canopy cover relative to the closed class (55 vs. 71\% mean canopy cover), lower tree heights (8.9 vs. $18.7 \mathrm{~m})$, lower tree basal area (9.1 vs. $20.7 \mathrm{~m}^{2} \mathrm{ha}^{-1}$ ), more stems (2291 vs. 2859 trees ha $^{-1}$ ) and smaller mean diameter at breast height $(\mathrm{dbh}=1.4 \mathrm{~m})(12 \mathrm{vs} .17$ $\mathrm{cm})$. Stand ages were not measured but the open canopy stands were typically younger successional stands in the temperate and tropical sites, and conversely have been older stands in the boreal sites. The open canopy stands in the boreal region (Alaska) were black spruce (Picea mariana [Mill.]) on cold soils underlain by permafrost and the closed canopy stands were dominated by white spruce (Picea glauca [Moench] Voss) on better drained soils not underlain by permafrost.

Three fragment size classes were selected based on field characterization and later classified and areas calculated using remote sensing software - see Meddens et al. (19), and by photo interpretation of a subset of the fragments as a component of an associated decomposition experiment - see González et al. (14). Overall mean fragment sizes were 104.5 ha (19.5 S.E.) for the large fragments, 23.1 ha (3.2 S.E.) for the medium fragments, and 10.9 ha $(0.5$ S.E.) for the small fragments.

Within these categories of climate, moisture, structure/age, and size we established a series of transects $(n=720)$ to sample from outside the forest fragment, across the edge, and into the forest interior (Fig. 2). Transects were located at accessible sites along the fragment edges and field observations and aerial photography were used to confirm fragment forest type and size categories. Along each transect we established plots at $0,10,20$, 40 , and $60 \mathrm{~m}$, with the forest boundary midway between 0 and $10 \mathrm{~m}$ (Fig. 2). In this analysis we analyzed plots only within the forested positions (positions 2-5) at 5, 15, 35, and 55 meters from the forest edge, respectively. At each plot we measured CWD and FWD, species composition, tree heights, diameters, crown dimensions, percent canopy cover, number of saplings, percent cover of shrubs, herbaceous material, litter, and bare ground, and litter and duff depths. CWD and FWD were measured using a line intercept method (20), and CWD and FWD biomass estimates were combined to determine downed woody debris (DWD) estimates in $\mathrm{Mg} \mathrm{ha}^{-1}$. Coarse woody debris was measured along $30 \mathrm{~m}$ transects and CWD diameters were measured and decay class estimated in one of five classes as CWD intersected the transect. Volume to mass conversions used values from Harmon and Sexton (20) for "unknown decay resistant species" for decay classes $1-5$. Fine woody debris was sampled in 3 classes: FWD1: 0.25 to $0.6 \mathrm{~cm}$, FWD2: 0.61 to 2.5 $\mathrm{cm}$, and FWD3: 2.51 to $7.6 \mathrm{~cm}$. These were tallied as they intersected transects of $3 \mathrm{~m}, 3 \mathrm{~m}$, and $6 \mathrm{~m}$ respectively per plot. Tallies of FWD were converted to biomass following Harmon and Sexton (20). Tree basal area was sampled using variable plots and basal area prisms. Tallied trees were identified to species and measured for dbh, height, height to crown, and crown width in two perpendicular directions. Snags were tallied similarly except for crown characteristics. Trees per hectare and biomass/ha were determined by assessing the relationship between basal area as estimated by the BAF prisms and the true basal area of a tree following a suggestion of Harmon and Sexton (20) and allometric equations of Scatena et al. (21). Litter and duff biomass were calculated from field measures of depth following Brown (22).

\section{Statistical Analyses}

Data were tested for homogeneity of variance by using Levene's test of equality of error variances, and skewness (23). A 5-way multivariate analysis of variance (MANOVA) was performed to determine the effect of climate (boreal, temperate, tropical), moisture (dry and moist forests), stand structure (open/young and closed/older), forest fragment size (small, medium, and large), and position along the forest edge gradient/transect (5, 15,35 , and $55 \mathrm{~m}$ from edge) on the forest floor biomass (litter and duff), DWD biomass, and live tree biomass. In addition, analyses of variance (ANOVA) were used to determine the effect of distance from forest edge (position) within climatic regions and among fragment sizes and structure, on structural, non living, and live fuels variables. Student-Newman-Keuls (SNK) tests were used to compare group means.

Edge effects: A significant effect of "position" indicated either significant differences between edge and interior plots, with a variable being either higher $(\uparrow)$ or lower $(\downarrow)$ as indicated in the results. Alternatively, the effect of position may indicate interior plots differing among themselves rather than indicating a clear cut edge effect. This situation is indicated as "mixed" (X) edge effects in the results. We performed linear and quadratic regressions on forest floor biomass (litter + duff), CWD, FWD, standing dead biomass (snags) and aboveground live tree biomass with mean annual median temperatures using all plots $(n=2529)$. Statistical analyses were performed using SPSS 16.0 (23).

\section{RESULTS}

\section{Regression Analyses}

We found a linear relationship of forest floor biomass (litter and duff combined) with mean annual median temperature (adj. 
Figure 3. Linear and quadratic relationships of forest floor fuels (litter and duff), CWD (open circles), FWD (filled squares), snags, and live tree biomass with mean annual median temperatures. Forest floor fuels increase linearly with temperature (and latitude) while dead woody debris and live biomass peak in temperate sites.
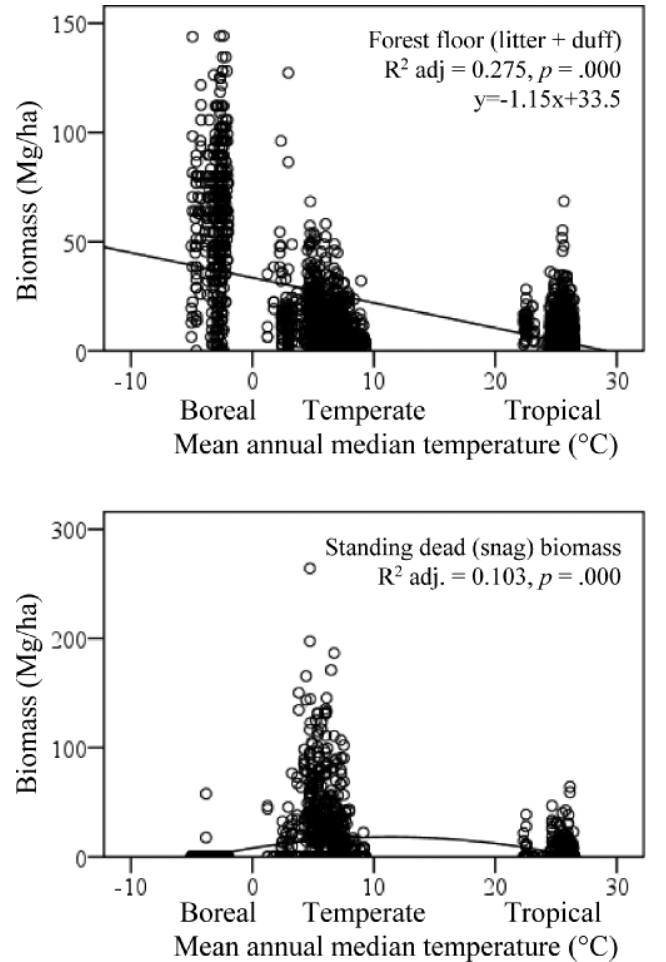
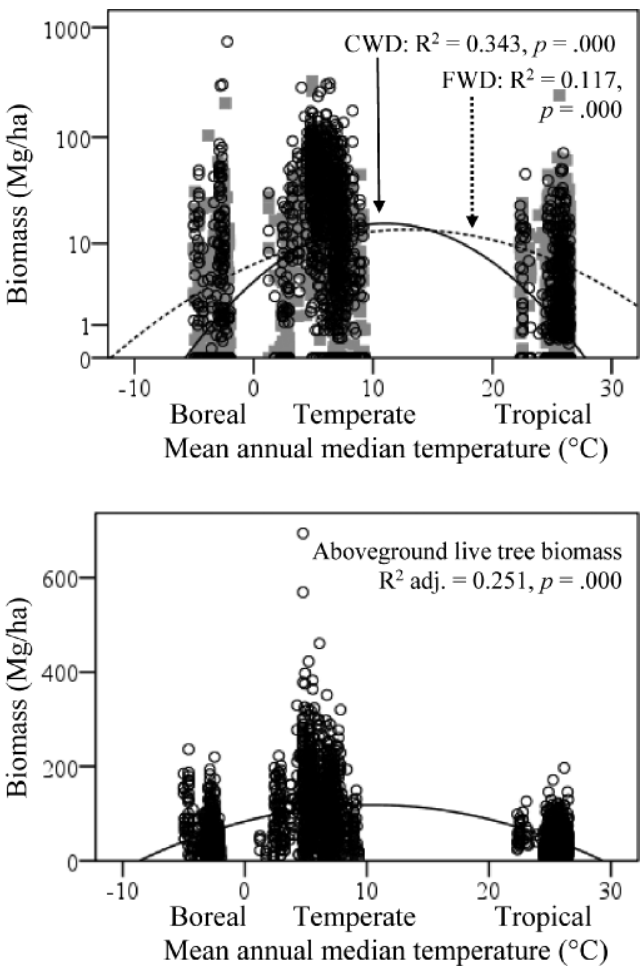

$\left.\mathrm{R}^{2}=0.275, p=0.000\right)$. Forest floor fuels were higher in the cooler climates. CWD, FWD, standing dead trees, and aboveground live tree biomass all showed nonlinear relationships with temperature. CWD had the strongest relationship (adj. $\left.\mathrm{R}^{2}=0.343, p=0.000\right)$ with a peak at approximately $10^{\circ} \mathrm{C}$ median annual temperature. FWD had a weaker relationship (adj. $\left.\mathrm{R}^{2}=0.117, p=0.000\right)$ and peaked at moderately warmer temperature. Standing dead tree (snag) biomass was greatest in the temperate region, as was live tree biomass $\left(\operatorname{adj} . \mathrm{R}^{2}=0.103\right.$ and 0.251 respectively, $p=0.000$ ) (Fig. 3).

\section{Forest Structure and Fuels}

We found significant effects of climate, moisture, structure, and fragment size on duff and litter biomass, DWD, and live tree biomass (Table 1). Canopy cover and mean tree dbh increased from boreal to tropical sites while mean tree height and basal area were greatest in temperate sites and least in tropical sites. The number of trees per hectare was significantly lower in temperate sites relative to boreal or tropical sites (Table 2). Fine woody debris was significantly greater in temperate sites, followed by tropical and then boreal sites (Table 2). Total DWD was greatest in temperate sites but did not differ between boreal and tropical sites (Table 2). Litter biomass increased from boreal to tropical sites and duff biomass decreased along the same climatic gradient and total forest floor biomass followed the same pattern (Table 2). The duff layer was the most significant fuel category in terms of biomass in the boreal sites, while CWD and snags were the most significant in the temperate sites. Fuels were more evenly spread among classes in the tropical sites but FWD class 3 (100 hour fuels) were relatively the most important (Table 2).

Table 1. Results from a five-way MANOVA for the effects of climate (C), moisture (M), forest age /structure (A), fragment size (S), and distance from edge or position (P) on the total biomass of duff and litter in the forest floor $\left(\mathrm{Mg} \mathrm{ha}^{-1}\right)$, total fine and coarse woody debris (Mg ha $\left.{ }^{-1}\right)$, and live tree aboveground biomass $\left(\mathrm{Mg} \mathrm{ha}^{-1}\right)$. Results not shown were not significant. Significance at $\alpha=0.05$ is indicated in bold, $\mathrm{N}=2454$.

\begin{tabular}{|c|c|c|c|c|c|c|c|c|c|c|}
\hline \multirow[b]{3}{*}{ Source } & \multirow[b]{3}{*}{ df } & \multicolumn{9}{|c|}{ Dependent variables } \\
\hline & & \multicolumn{3}{|c|}{ Duff and litter biomass* } & \multicolumn{3}{|c|}{ Downed woody debris ${ }^{\dagger}$} & \multicolumn{3}{|c|}{ Live tree biomass } \\
\hline & & $\mathbf{F}$ & Sig. & Power & $\mathbf{F}$ & Sig. & Power & $\mathbf{F}$ & Sig. & Power \\
\hline Climate (C) & 2 & 387.85 & 0.00 & 1.00 & 97.74 & 0.00 & 1.00 & 166.53 & 0.00 & 1.00 \\
\hline Moisture (M) & 1 & 226.60 & 0.00 & 1.00 & 27.60 & 0.00 & 1.00 & 111.28 & 0.00 & 1.00 \\
\hline Age/structure $(A)$ & 1 & 6.45 & 0.01 & 0.72 & 14.29 & 0.00 & 0.97 & 377.95 & 0.00 & 1.00 \\
\hline Size (S) & 2 & 5.82 & 0.00 & 0.87 & 0.26 & 0.77 & 0.09 & 2.49 & 0.08 & 0.50 \\
\hline Position (P) & 3 & 1.01 & 0.39 & 0.28 & 0.01 & 1.00 & 0.05 & 1.62 & 0.18 & 0.43 \\
\hline $\mathrm{C} * \mathrm{M}$ & 2 & 242.66 & 0.00 & 1.00 & 36.41 & 0.00 & 1.00 & 11.76 & 0.00 & 0.99 \\
\hline$C * A$ & 2 & 21.21 & 0.00 & 1.00 & 11.19 & 0.00 & 0.99 & 157.38 & 0.00 & 1.00 \\
\hline$C * S$ & 4 & 5.67 & 0.00 & 0.98 & 0.13 & 0.97 & 0.08 & 3.41 & 0.01 & 0.86 \\
\hline$C * P$ & 6 & 0.96 & 0.45 & 0.39 & 0.75 & 0.61 & 0.30 & 0.30 & 0.93 & 0.14 \\
\hline$M * A$ & 1 & 2.60 & 0.11 & 0.36 & 0.18 & 0.67 & 0.07 & 27.61 & 0.00 & 1.00 \\
\hline$M * S$ & 2 & 0.85 & 0.43 & 0.20 & 1.56 & 0.21 & 0.33 & 0.71 & 0.49 & 0.17 \\
\hline$M * P$ & 3 & 0.85 & 0.47 & 0.24 & 0.44 & 0.72 & 0.14 & 0.75 & 0.52 & 0.21 \\
\hline$A * S$ & 2 & 1.45 & 0.23 & 0.31 & 1.92 & 0.15 & 0.40 & 0.71 & 0.49 & 0.17 \\
\hline$A * P$ & 3 & 0.77 & 0.51 & 0.22 & 0.35 & 0.79 & 0.12 & 0.33 & 0.80 & 0.11 \\
\hline$S * P$ & 6 & 1.00 & 0.42 & 0.40 & 0.04 & 1.00 & 0.06 & 0.57 & 0.75 & 0.23 \\
\hline$C{ }^{*} M * A$ & 2 & 3.96 & 0.02 & 0.71 & 2.22 & 0.11 & 0.46 & 10.23 & 0.00 & 0.99 \\
\hline Error & 2310 & & & & & & & & & \\
\hline
\end{tabular}


Table 2. Mean and standard deviation for climatic factors, structural characteristics, non living and living fuels for sites in boreal, temperate, and tropical environments. Statistical differences among means of each characteristic are given by letters. Edge effect is indicated as $\uparrow$ or $\downarrow$ if variable was significantly higher or lower respectively in edge position or as " $X$ " if the effect of position was significant but with a mixed effect between edge and interior plots. Climatic factors were not tested for edge effect.

\begin{tabular}{ccccc} 
Boreal & & & \multicolumn{3}{c}{ Temperate } \\
\cline { 5 - 5 } \cline { 4 - 4 } S.D. & $\begin{array}{c}\text { Edge } \\
\text { effect }\end{array}$ & & $\begin{array}{l}\text { Edge } \\
\text { effect }\end{array}$
\end{tabular}

\begin{tabular}{cc} 
Tropical & \\
\hline S.D. & $\begin{array}{c}\text { Edge } \\
\text { effect }\end{array}$
\end{tabular}

\section{Climatic factors}

Mean annual precipitation $(\mathrm{mm})$

Mean annual median temperature $\left({ }^{\circ} \mathrm{C}\right)$

Mean latitude ( ${ }^{\circ}$ North)

Number of plots

$\begin{array}{cr}407 & 137 \\ -2.0 & 2.1 \\ 62.3 & 5.9\end{array}$

2.1
5.9

$\begin{array}{rr}922 & 265 \\ 6.3 & 1.2 \\ 46.9 & 1.7 \\ 1027 & \end{array}$

1027

7

1668

25.6

493

Structural aspects

Dominant vegetation

Coniferous evergreen

Canopy cover (\%)

Tree height $(\mathrm{m})$

Tree basal area $\left(\mathrm{m}^{2} \mathrm{ha}^{-1}\right)$

Number of trees per hectare

Mean Dbh of trees (cm)

$\begin{array}{rrr}52.0^{\mathrm{a}} & 28.3 & - \\ 11.3^{\mathrm{a}} & 7.3 & - \\ 14.3^{\mathrm{a}} & 10.1 & - \\ 3194.4^{\mathrm{a}} & 4215.6 & - \\ 13.3^{\mathrm{a}} & 8.7 & -\end{array}$

Coniferous evergreen

Non living fuels

1 hour fuels (FWD1) $\left(\mathrm{Mg} \mathrm{ha}^{-1}\right)$

10 hour fuels (FWD2) $\left(\mathrm{Mg} \mathrm{ha}^{-1}\right)$

100 hour fuels (FWD3) (Mg ha ${ }^{-1}$ )

Total FWD (Mg ha $\left.{ }^{-1}\right)$

1000 hour fuels (CWD) $\left(\mathrm{Mg} \mathrm{ha}^{-1}\right)$

Total downed woody debris (Mg ha ${ }^{-1}$ )

Snags $\left(\mathrm{Mg} \mathrm{ha}^{-1}\right)$

Total dead woody debris $\left(\mathrm{Mg} \mathrm{ha}^{-1}\right)$

Litter biomass ( $\mathrm{Mg} \mathrm{ha}^{-1}$ )

Duff biomass $\left(\mathrm{Mg} \mathrm{ha}^{-1}\right)$

Forest floor biomass $\left(\mathrm{Mg} \mathrm{ha}^{-1}\right)$

$\begin{array}{ll}0.5^{\mathrm{a}} & 0.9 \\ 1.7^{a} & 3.3\end{array}$

$\begin{array}{ll}0.5^{\mathrm{a}} & 0.9 \\ 1.7^{\text {a }} & 3.3 \\ 4.2^{\mathrm{a}} & 9.8\end{array}$

$4.2^{\mathrm{a}} \quad 9.8$

$6.5^{\mathrm{a}} \quad 12.2$

$7.0^{\mathrm{a}} \quad 38.3$

$13.5^{\mathrm{a}} \quad 40.4$

$0.8^{\mathrm{a}} \quad 5.0$

$14.3^{\text {a }} \quad 40.9$

$0.7^{\mathrm{a}} \quad 1.6$

$53.0^{\mathrm{a}} \quad 33.8$

$53.7^{\mathrm{a}} \quad 33.1$

Live fuels

Tree biomass $\left(\mathrm{Mg} \mathrm{ha}^{-1}\right)$

Shrub cover (\%)

Herbaceous cover (\%)

$\begin{array}{lll}60.1^{\mathrm{a}} & 47.6 & - \\ 32.5^{\mathrm{a}} & 26.5 & - \\ 17.4^{\mathrm{a}} & 23.6 & -\end{array}$

$\begin{array}{rrr}74.5^{\mathrm{b}} & 21.9 & \mathrm{X} \\ 22.2^{\mathrm{b}} & 7.3 & - \\ 27.4^{\mathrm{b}} & 17.0 & - \\ 2572.6^{\mathrm{b}} & 2002.3 & - \\ 14.9^{\mathrm{b}} & 5.5 & -\end{array}$

960

Broadleaf evergreen and semideciduous

\begin{tabular}{rrr}
\hline $81.7^{\mathrm{c}}$ & 23.4 & $\mathrm{X}$ \\
$7.4^{\mathrm{c}}$ & 2.7 & $\mathrm{X}$ \\
$10.4^{\mathrm{c}}$ & 5.9 & $\mathrm{X}$ \\
$3331.9^{\mathrm{a}}$ & 5263.8 & - \\
$15.2^{\mathrm{b}}$ & 10.8 & -
\end{tabular}

$\begin{array}{rrr}1.5^{\mathrm{b}} & 1.3 & - \\ 5.8^{\mathrm{b}} & 5.5 & - \\ 10.8^{\mathrm{b}} & 15.8 & - \\ 18.0^{\mathrm{b}} & 18.6 & - \\ 31.2^{\mathrm{b}} & 37.1 & - \\ \mathbf{4 9 . 2 ^ { \mathrm { b } }} & 46.3 & - \\ 17.6^{\mathrm{b}} & 31.0 & - \\ \mathbf{6 6 . 8 ^ { \mathrm { b } }} & 64.4 & - \\ 3.1^{\mathrm{b}} & 2.1 & - \\ 8.9^{\mathrm{b}} & 9.3 & - \\ 11.9^{\mathrm{b}} & 10.1 & - \\ & & \\ 116.3^{\mathrm{b}} & 77.7 & - \\ 23.1^{\mathrm{b}} & 23.1 & - \\ 33.6^{\mathrm{b}} & 28.4 & -\end{array}$

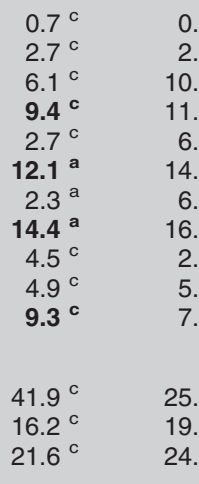

Live tree biomass was significantly greater in temperate followed by boreal and tropical sites (Table 2). Live biomass was greater in moist vs. dry sites and closed/older vs. open/ younger forest stands in all biomes (Figs. 4, 5). Open forest stands were not significantly different in live biomass among boreal, temperate, and tropical sites (Fig. 5). The relative distribution of dead fuels varied considerably among climates

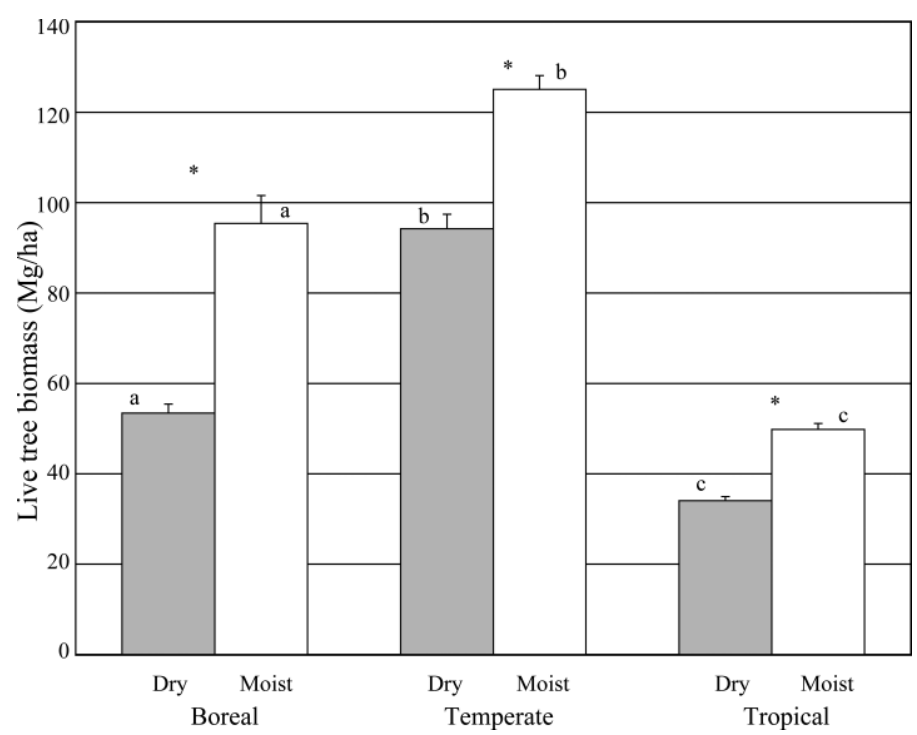

Figure 4. Variation in live tree biomass along the climatic gradient and between dry and moist forests. Asterisks $\left(^{*}\right)$ indicate significant differences within a climatic region, letters indicate significant differences among dry or moist sites along the climatic gradient $(\alpha=0.05$, error bars as S.E). and was strongly controlled by moisture (Table 3). Boreal dry sites had the greatest component of duff layer biomass, while temperate moist sites had the greatest component of CWD and standing dead snags (Fig. 6). Fine woody debris was greatest in temperate moist sites but showed the least variability among biomes (Table 3, Fig. 6). Total live and dead aboveground biomass ranged from $53.9 \mathrm{Mg} \mathrm{ha}^{-1}$ in the tropical dry sites to

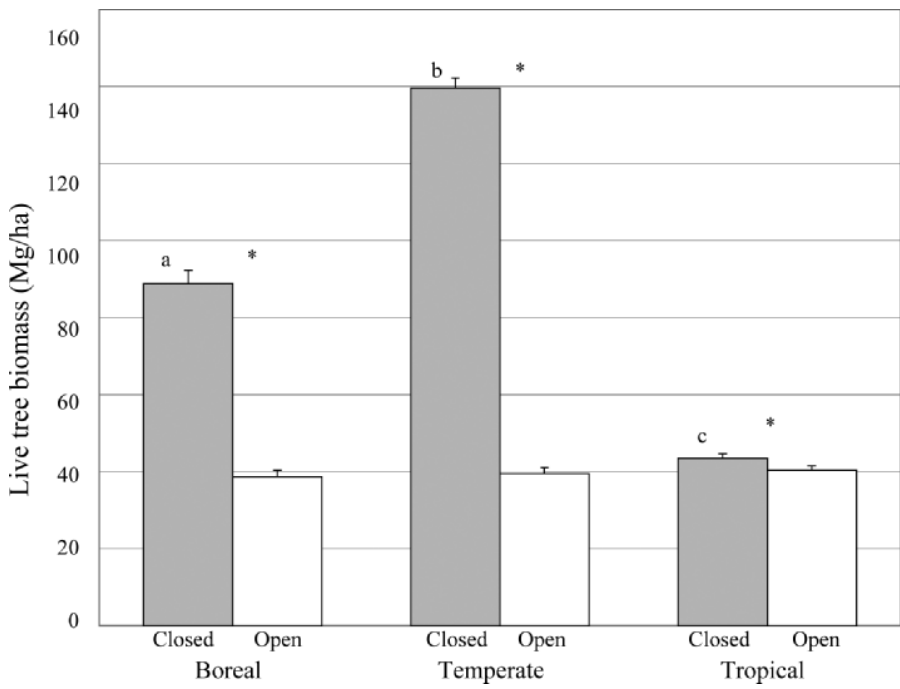

Figure 5. Variation in live tree biomass along the climatic gradient and between closed/older and open/younger forest stands. Asterisks $\left(^{*}\right)$ indicate significant differences within a climatic region, letters indicate significant differences among closed or open sites along the climatic gradient $(\alpha=0.05$, error bars as S.E). Open/ younger stands were not significantly different along the climatic gradient. 
Table 3. Mean and standard deviation for climatic factors, structural characteristics, non living and living fuels for sites in dry and moist forest fragments in boreal, temperate, and tropical environments. Statistical differences between means of each characteristic within each climatic region are given by letters. Edge effect is indicated as $\uparrow$ or $\downarrow$ if variable was significantly higher or lower respectively in edge position or as " $X$ " if the effect of position was significant but with a mixed effect between edge and interior plots. Climatic factors were not tested for edge effect.

\begin{tabular}{|c|c|c|c|c|c|c|c|}
\hline \multicolumn{3}{|c|}{ Boreal } & & \multicolumn{4}{|c|}{ Temperate } \\
\hline Dry & & Moist & & Dry & & Moist & \\
\hline \pm S.D. & $\begin{array}{l}\text { Edge } \\
\text { effect }\end{array}$ & \pm S.D. & $\begin{array}{l}\text { Edge } \\
\text { effect }\end{array}$ & \pm S.D. & $\begin{array}{l}\text { Edge } \\
\text { effect }\end{array}$ & \pm S.D. & $\begin{array}{l}\text { Edge } \\
\text { effect }\end{array}$ \\
\hline
\end{tabular}

\section{Climatic factors}

Mean annual precipitation $(\mathrm{mm})$

(degrees North)

Number of plots

$\begin{array}{rr}359.3 & 74.1 \\ -2.9 & 1.0 \\ 64.6 & 0.8 \\ 461.0 & \\ & \\ 49.9^{\mathrm{a}} & 28.9 \\ 10.2^{\mathrm{a}} & 6.7 \\ 12.4^{\mathrm{a}} & 8.2 \\ 3061.5^{\mathrm{a}} & 4285.3 \\ 13.7^{\mathrm{a}} & 9.2\end{array}$

ructural aspects

Canopy cover (percent)

Tree height $(\mathrm{m})$

Tree basal area $\left(\mathrm{m}^{2} \mathrm{ha}^{-1}\right)$

Trees per hectare

Mean Dbh of trees $(\mathrm{cm})$

$13.7^{a}$

Non living fuels

1 hour fuels (FWD1)

10 hour fuels (FWD2)

100 hour fuels (FWD3)

Total FWD (Mg ha ${ }^{-1}$ )

0.6

1000 hour fuels (CWD) (Mg ha ${ }^{-1}$

$1.7^{\mathrm{a}}$

$4.6^{\mathrm{a}}$

$6.9^{\mathrm{a}}$

Total downed woody debris $\left(\mathrm{Mg} \mathrm{ha}^{-1}\right)$

$7.6^{\mathrm{a}}$

Snags $\left(\mathrm{Mg} \mathrm{ha}^{-1}\right)$

Total dead woody debris $\left(\mathrm{Mg} \mathrm{ha}^{-1}\right)$

$14.5^{\mathrm{a}}$

$0.4^{\mathrm{a}}$
$14.8^{\mathrm{a}}$

Litter biomass ( $\mathrm{Mg} \mathrm{ha}^{-1}$ )

Duff biomass ( $\mathrm{Mg} \mathrm{ha}^{-1}$ )

Forest floor biomass $\left(\mathrm{Mg} \mathrm{ha}^{-1}\right)$

$14.8^{\mathrm{a}}$
$0.2^{\mathrm{a}}$

$0.2^{\mathrm{a}}$
$60.0^{\mathrm{a}}$

$60.0^{\mathrm{a}}$
$60.2^{\mathrm{a}}$

0.9
3.5

3.5
10.5

13.0

41.5

43.6

4.1

44.1

0.7
31.0

31.0
30.9

Live fuels

Tree biomass $\left(\mathrm{Mg} \mathrm{ha}^{-1}\right)$

Shrub cover (percent)

53.4

Herbaceous cover (percent)

$35.9^{\mathrm{a}}$

$13.0^{\mathrm{a}}$

668.0

2.4

2.4
49.5
83.0

1.3

5.2

682.4

7.2
44.8

1.1

1.8

1016.7

230.5

292.0

47.8

736.0

$616^{a}$

$\begin{array}{lr}63.9^{b} & 21.1 \\ 17.2^{\mathrm{b}} & 7.4\end{array}$

$24.7^{\mathrm{b}} \quad 12.9$

$3917.8^{\mathrm{b}} \quad 3755.6$

$\begin{array}{lr}11.2^{\mathrm{b}} & 4.7\end{array}$

$21.6^{\mathrm{a}}$

$222^{\mathrm{a}}$

13.1

$1915.5^{\mathrm{a}} \quad 1715.3$

$\begin{array}{ll}15.7^{\mathrm{a}} & 5.3\end{array}$

$0.8^{\mathrm{a}} \quad 1.0$

$\begin{array}{ll}4.1^{\mathrm{a}} & 4.9\end{array}$

$\begin{array}{ll}7.1^{\mathrm{a}} & 8.3\end{array}$

$12.0^{\mathrm{a}} \quad 11.2$

$13.3^{\mathrm{a}} \quad 28.0$

$25.3^{\mathrm{a}} \quad 33.6$

$4.2^{\mathrm{a}} \quad 10.0$

$29.5^{\mathrm{a}} \quad 38.1$

$3.7^{\mathrm{a}}$

$6.2^{\mathrm{a}}$

$9.9^{\mathrm{a}}$

$15.1^{\mathrm{b}}$

$18.5^{\mathrm{b}}$

2.5
20.6

19.7

2.4

6.3

$94.2^{\mathrm{a}}$

$25.7^{a}$

$39.6^{\mathrm{a}}$

\section{4}

23.8

27.6
29.5
0.6

0.5
1.1
0.6



19.8
7.7
17.8
48.8
5.5

1.3

5.6

17.7

20.3

37.9

47.2

34.7

66.7

1.8

10.1

10.7

$\begin{array}{llll}42.0 & - & 14.2^{\mathrm{b}} & 21.6 \\ 19.3 & - & 41.4^{\mathrm{b}} & 29.5\end{array}$

27.6

\begin{tabular}{l}
$41.4^{\mathrm{b}} \quad 29.5$ \\
\hline
\end{tabular}

(20)




\begin{tabular}{|c|c|c|c|c|c|}
\hline \multicolumn{6}{|c|}{ Tropical } \\
\hline \multicolumn{3}{|c|}{ Dry } & \multicolumn{3}{|c|}{ Moist } \\
\hline & \pm S.D. & $\begin{array}{l}\text { Edge } \\
\text { effect }\end{array}$ & & \pm S.D. & $\begin{array}{l}\text { Edge } \\
\text { effect }\end{array}$ \\
\hline 1328 & 359.0 & & 1992 & 370.9 & n.m. \\
\hline 25.8 & 0.6 & & 25.4 & 0.9 & n.m. \\
\hline 18.1 & 0.2 & & 18.3 & 0.1 & n.m. \\
\hline 489.0 & & & 471.0 & & \\
\hline $76.3^{\mathrm{a}}$ & 26.0 & - & $87.4^{b}$ & 18.6 & $\uparrow$ \\
\hline $6.1^{\mathrm{a}}$ & 1.9 & - & $8.7^{\mathrm{b}}$ & 2.7 & $x$ \\
\hline $9.6^{a}$ & 5.7 & - & $11.3^{\mathrm{b}}$ & 6.0 & $\downarrow$ \\
\hline $4008.7^{a}$ & 5945.2 & - & $2629.3^{\mathrm{b}}$ & 4343.4 & - \\
\hline $11.4^{\mathrm{a}}$ & 8.1 & - & $19.0^{\mathrm{b}}$ & 11.8 & - \\
\hline $0.6^{a}$ & 0.4 & - & $0.8^{\mathrm{b}}$ & 0.6 & - \\
\hline $2.4^{\mathrm{a}}$ & 2.4 & - & $2.9^{\mathrm{b}}$ & 3.0 & - \\
\hline $5.2^{\mathrm{a}}$ & 6.8 & - & $7.0^{\mathrm{b}}$ & 13.4 & - \\
\hline $8.3^{a}$ & 7.7 & - & $10.6^{\mathrm{b}}$ & 14.5 & - \\
\hline $1.4^{\mathrm{a}}$ & 3.8 & - & $4.0^{\mathrm{b}}$ & 8.0 & - \\
\hline $9.7^{\mathrm{a}}$ & 9.1 & - & $14.6^{\mathrm{b}}$ & 17.5 & - \\
\hline $1.7^{\mathrm{a}}$ & 4.4 & - & $2.9^{\mathrm{b}}$ & 7.9 & - \\
\hline $11.5^{\mathrm{a}}$ & 10.3 & - & $17.5^{\mathrm{b}}$ & 20.5 & - \\
\hline $4.0^{\mathrm{a}}$ & 2.4 & - & $4.9^{\mathrm{b}}$ & 2.8 & - \\
\hline $4.4^{\mathrm{a}}$ & 5.1 & - & $5.4^{\mathrm{b}}$ & 6.3 & - \\
\hline $8.4^{\mathrm{a}}$ & 6.5 & - & $10.3^{b}$ & 8.0 & - \\
\hline $34.0^{\mathrm{a}}$ & 20.0 & - & $49.8^{b}$ & 28.3 & $x$ \\
\hline $16.5^{\mathrm{a}}$ & 19.2 & - & $15.8^{\mathrm{b}}$ & 19.5 & 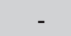 \\
\hline $21.2^{\mathrm{a}}$ & 26.3 & - & $21.9^{\mathrm{b}}$ & 22.8 & - \\
\hline
\end{tabular}

Table 4. Extended.

\begin{tabular}{|c|c|c|c|c|c|}
\hline \multicolumn{6}{|c|}{ Tropical } \\
\hline \multicolumn{3}{|c|}{ Open } & \multicolumn{3}{|c|}{ Closed } \\
\hline & \pm S.D. & $\begin{array}{l}\text { Edge } \\
\text { effect }\end{array}$ & & \pm S.D. & $\begin{array}{l}\text { Edge } \\
\text { effect }\end{array}$ \\
\hline $78.3^{\mathrm{a}}$ & 25.2 & $\mathrm{x}$ & $85.4^{\mathrm{b}}$ & 20.7 & - \\
\hline $7.0^{\mathrm{a}}$ & 2.6 & - & $7.8^{\mathrm{b}}$ & 2.7 & $x$ \\
\hline $10.3^{\mathrm{a}}$ & 6.1 & - & $10.6^{\mathrm{a}}$ & 5.6 & $\hat{x}$ \\
\hline $3580.6^{\mathrm{a}}$ & 6592.8 & - & $3061.6^{a}$ & 3239.7 & $x$ \\
\hline $14.6^{\mathrm{a}}$ & 10.8 & - & $15.8^{\mathrm{a}}$ & 10.7 & - \\
\hline $0.7^{\mathrm{a}}$ & 0.6 & - & $0.7^{b}$ & 0.5 & - \\
\hline $2.7^{\mathrm{a}}$ & 2.8 & - & $2.6^{a}$ & 2.5 & - \\
\hline $6.1^{\mathrm{a}}$ & 12.6 & - & $6.1^{\mathrm{a}}$ & 7.8 & - \\
\hline $9.5^{\mathrm{a}}$ & 13.7 & - & $9.4^{\mathrm{a}}$ & 8.8 & - \\
\hline $2.4^{\mathrm{a}}$ & 5.5 & - & $3.0^{\mathrm{a}}$ & 7.2 & - \\
\hline $11.9^{\mathrm{a}}$ & 15.6 & - & $12.4^{\mathrm{a}}$ & 12.2 & $x$ \\
\hline $2.3^{\mathrm{a}}$ & 6.0 & - & $2.2^{\mathrm{a}}$ & 6.8 & - \\
\hline $14.2^{\mathrm{a}}$ & 17.8 & - & $14.7^{\mathrm{a}}$ & 14.7 & $x$ \\
\hline $4.9^{\mathrm{a}}$ & 2.6 & - & $4.1^{\mathrm{b}}$ & 2.6 & - \\
\hline $5.6^{\mathrm{a}}$ & 6.5 & - & $4.2^{\mathrm{b}}$ & 4.9 & - \\
\hline $10.5^{\mathrm{a}}$ & 8.0 & - & $8.3^{b}$ & 6.5 & - \\
\hline $40.4^{a}$ & 26.1 & - & $43.5^{\mathrm{a}}$ & 25.1 & $\mathrm{x}$ \\
\hline $17.1^{\mathrm{a}}$ & 19.1 & - & $15.1^{\mathrm{a}}$ & 19.6 & - \\
\hline $23.7^{\mathrm{a}}$ & 26.2 & - & $19.2^{\mathrm{b}}$ & 22.7 & - \\
\hline
\end{tabular}

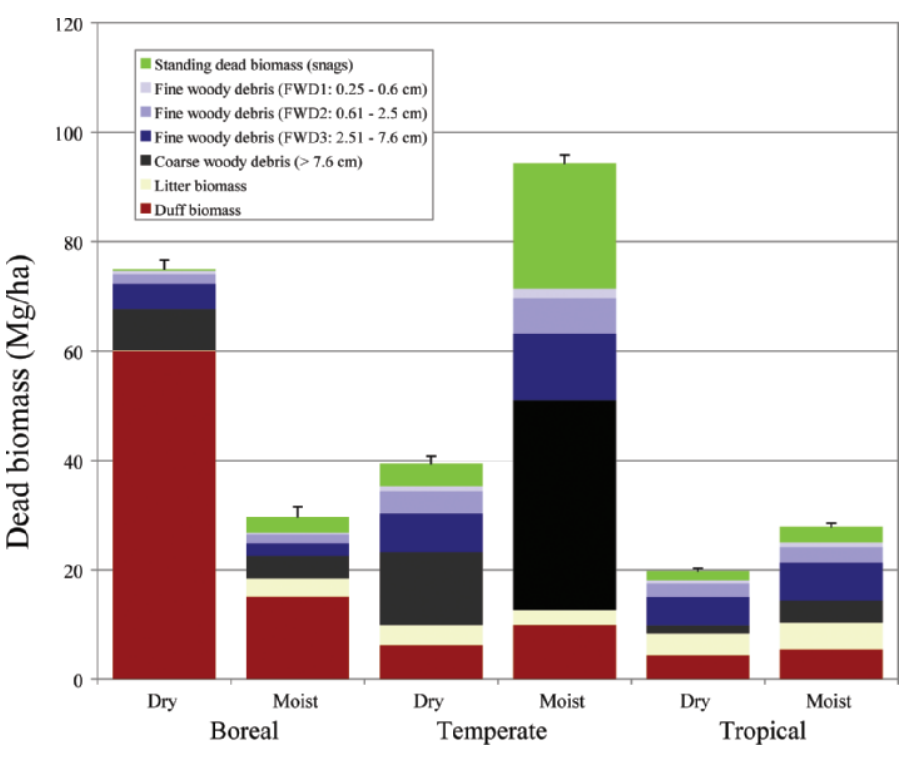

Figure 6. Downed fuels, litter, duff and standing dead fuels for dry and moist forest types along the climatic gradient. $(\alpha=0.05$, error bars as S.E). Dry and moist sites are significantly different in all climate regions.

219.4 $\mathrm{Mg} \mathrm{ha}^{-1}$ in the temperate moist sites. The greatest component of above ground biomass was the duff layer in the boreal dry sites and the live tree biomass in all other sites.

Closed canopy/older forests had significantly greater canopy cover, tree height, basal area, and mean tree dbh and fewer trees per hectare than open canopy/younger forests in boreal and temperate sites. Open and closed canopy forests were more similar in tropical sites but differed significantly in canopy cover and tree height (Table 4). In the temperate sites, closed canopy forests had significantly greater dead woody fuels in all categories except litter and FWD2, which did not differ by structure. Boreal forests had significantly greater amounts of FWD and forest floor biomass in closed sites than in open sites. Nearly all dead woody fuels and forest floor biomass were significantly different between temperate open and closed sites. In contrast, downed woody fuels were not significantly different between open and closed canopy sites in tropical forests, with the exception of slightly less litter and duff in closed canopy forest sites (Table 4). Herbaceous cover and shrub cover were greater in open $v s$. closed canopy sites in temperate and tropical forests while herbaceous cover was greater in closed vs. open canopy forests (Table 4).

Total dead woody debris was significantly greater in large fragments $v s$. medium and small fragments in the temperate and tropical sites. A similar but nonsignificant pattern was seen in the boreal sites. Smaller fragments had higher live tree biomass than large fragments in the boreal and temperate sites (Table 5).

\section{Edge Effects}

While no consistent edge effects were seen that crossed all climate, moisture, or structural categories, we saw a number of edge effects within subcategories. In terms of canopy cover, no differences related to position were seen in the boreal sites and the temperate and tropical sites had mixed effects, with a consistent drop in canopy cover at the most interior position (Table 2, Fig. 7a). Tree heights and basal area showed similar mixed effects in the tropical sites. Tree biomass showed mixed effects in the tropical sites (Fig. 7b) while the percent of herbaceous cover was significantly greater in the edge vs. interior plots (Table 2, Fig. 7c). Moist temperate sites showed the same pattern as temperate sites (moist and dry combined), with mixed effects related to position. Tropical moist sites 
Table 5. Mean and standard deviation for climatic factors, structural characteristics, non living and living fuels for sites in large, medium, and small forest fragments in boreal, temperate, and tropical environments. Statistical differences among means of each characteristic within each climatic region are given by letters. Edge effect is indicated as $\uparrow$ or $\downarrow$ if variable was significantly higher or lower respectively in edge position or as " $X$ " if the effect of position was significant but with a mixed effect between edge and interior plot.

\begin{tabular}{|c|c|c|c|c|c|c|c|c|c|c|c|c|}
\hline & \multicolumn{8}{|c|}{ Boreal } & & \multirow{2}{*}{\multicolumn{2}{|c|}{$\begin{array}{c}\text { Temperate } \\
\text { Large }\end{array}$}} & \\
\hline & \multicolumn{3}{|c|}{ Large } & \multicolumn{3}{|c|}{ Medium } & \multicolumn{3}{|c|}{ Small } & & & \\
\hline & \multicolumn{3}{|c|}{$\begin{array}{l}\text { Edge } \\
\text { effect }\end{array}$} & \multicolumn{3}{|c|}{$\begin{array}{l}\text { Edge } \\
\text { effect }\end{array}$} & \multicolumn{3}{|c|}{$\begin{array}{l}\text { Edge } \\
\text { effect }\end{array}$} & \multicolumn{3}{|c|}{$\begin{array}{l}\text { Edge } \\
\text { effect }\end{array}$} \\
\hline \multicolumn{13}{|l|}{ Structural characteristics } \\
\hline Canopy cover $(\%)$ & $48.1^{\mathrm{a}}$ & 27.4 & - & $53.7^{\mathrm{a}}$ & $27.9-$ & - & $53.3^{\mathrm{a}}$ & 28.9 & - & $75.4^{\mathrm{a}}$ & 23.- & - \\
\hline Tree height $(\mathrm{m})$ & $9.3^{\mathrm{a}}$ & 6.5 & - & $11.9^{\mathrm{b}}$ & $7.0-$ & - & $12.1^{\mathrm{b}}$ & 7.7 & - & $22.0^{a}$ & 6.6 & - \\
\hline Tree basal area $\left(\mathrm{m}^{2} \mathrm{ha}^{-1}\right)$ & $13.2^{\mathrm{a}}$ & 10.3 & - & $14.7^{\mathrm{a}}$ & 10.4 & - & 14.8 & 9.8 & - & $28.1^{\mathrm{a}}$ & 17.6 & - \\
\hline Trees per hectare & $3956.9^{a}$ & 5017.8 & - & $2942.6^{\mathrm{b}}$ & 4147.1 & - & $2900.3^{\mathrm{b}}$ & 3640.4 & - & $2628.7^{a}$ & 1966.7 & - \\
\hline Mean Dbh of trees $(\mathrm{cm})$ & $10.4^{a}$ & 5.4 & - & $13.7^{\mathrm{b}}$ & 8.4 & & $14.8^{\mathrm{b}}$ & 9.9 & - & $14.4^{a}$ & 4.8 & - \\
\hline \multicolumn{13}{|l|}{ Non living fuels } \\
\hline 1 hour fuels (FWD1) & $0.7^{\mathrm{a}}$ & 1.2 & - & $0.6^{\mathrm{a}}$ & 0.8 & & $0.5^{\mathrm{a}}$ & 0.6 & - & $1.6^{\mathrm{a}}$ & 1.4 & - \\
\hline 10 hour fuels (FWD2) & $1.7^{\mathrm{a}}$ & 1.8 & - & $1.5^{\mathrm{a}}$ & 1.9 & - & $1.9^{\mathrm{a}}$ & 4.5 & - & $5.6^{\mathrm{a}}$ & 5.1 & - \\
\hline 100 hour fuels (FWD3) & $3.6^{a}$ & 6.4 & - & $3.7^{\mathrm{a}}$ & 7.3 & - & $5.0^{\mathrm{a}}$ & 12.8 & - & $11.7^{\mathrm{a}}$ & 17.1 & - \\
\hline Total FWD ( $\left.\mathrm{Mg} \mathrm{ha}^{-1}\right)$ & $6.0^{\mathrm{a}}$ & 7.0 & - & $5.8^{\mathrm{a}}$ & 8.2 & - & $7.3^{\mathrm{a}}$ & 16.4 & - & $18.9^{a}$ & 19.6 & - \\
\hline 1000 hour fuels (CWD) (Mg ha- 1 ) & $9.4^{\mathrm{a}}$ & 66.7 & - & $8.1^{\mathrm{a}}$ & 27.1 & - & $4.8^{\mathrm{a}}$ & 11.9 & - & $35.8^{a}$ & 39.7 & - \\
\hline Total downed woody debris (Mg ha-1) & $15.4^{\mathrm{a}}$ & 66.6 & - & $13.9^{\mathrm{a}}$ & 29.5 & - & $12.1^{\mathrm{a}}$ & 20.9 & - & $54.7^{\mathrm{a}}$ & 49.5 & - \\
\hline Snags (Mg ha- 1 ) & $1.0^{\mathrm{a}}$ & 5.4 & - & $0.5^{\mathrm{a}}$ & 3.1 & - & $0.8^{\mathrm{a}}$ & 5.8 & - & $22.5^{\mathrm{a}}$ & 33.5 & - \\
\hline Total dead woody debris (Mg ha-1) & $16.4^{\mathrm{a}}$ & 66.8 & - & $14.4^{\mathrm{a}}$ & 29.7 & - & $12.9^{\mathrm{a}}$ & 22.9 & - & $77.4^{\mathrm{a}}$ & 71.1 & - \\
\hline Litter biomass (Mg ha-1) & $0.9^{a}$ & 2.1 & - & $0.8^{\mathrm{a}}$ & 1.6 & - & $0.5^{\mathrm{a}}$ & 1.3 & & $2.8^{\mathrm{a}}$ & 1.9 & \\
\hline Duff biomass (Mg ha-1) & $58.4^{a}$ & 32.1 & - & $50.4^{\mathrm{a}}$ & 33.4 & & $51.5^{\mathrm{a}}$ & 34.8 & - & $9.2^{\mathrm{a}}$ & 9.9 & - \\
\hline Forest floor biomass (Mg ha-1) & $59.3^{\mathrm{a}}$ & 30.8 & - & $51.1^{\mathrm{a}}$ & 32.7 & - & $52.1^{\mathrm{a}}$ & 34.4 & - & $12.0^{\text {ab }}$ & 10.6 & - \\
\hline Live fuels & & & & & & - & & & - & & & \\
\hline Tree biomass (Mg ha- 1 ) & $50.9^{a}$ & 42.4 & & $61.4^{b}$ & 46.3 & - & $64.7^{\mathrm{b}}$ & 50.8 & - & $119.3^{a}$ & 78.2 & - \\
\hline Shrub cover $(\%)$ & $33.7^{\mathrm{a}}$ & 28.5 & - & $33.9^{\mathrm{a}}$ & 27.1 & - & $30.9^{a}$ & 24.8 & - & $22.6^{a}$ & 22.6 & - \\
\hline Herbaceous cover (\%) & $17.2^{\mathrm{a}}$ & 22.5 & - & $20.3^{a}$ & 25.7 & - & $15.5^{\mathrm{a}}$ & 22.5 & - & $34.9^{\mathrm{a}}$ & 29.5 & - \\
\hline
\end{tabular}

showed an increase in canopy cover and a decrease in basal area at forest edges, with mixed effects of tree height and tree biomass (Table 3). Temperate open forest sites showed greater canopy cover at forest edges, while tropical open sites showed mixed effects. Tropical closed forest sites showed mixed effects of canopy height, basal area, number of tree stems, total DWD, total dead wood (Fig. 7d), and tree biomass (Table 4). Little edge effect was related to fragment size aside from a mixed effect of position in small temperate fragments (Table 5).

\section{DISCUSSION}

The goal of this study was to look at the relationship between forest structural characteristics, woody debris fuels, and forest fragmentation. We assessed the relative value of structural and fuels variables and distance from forest edge in a wide range of forest fragment types to better understand the variability and type of fuels associated with various components of a fragmented landscape. To do that we assessed forest fuel
Figure 7. Temperate (grey bars) and tropical (white bars) sites show patterns of a mixed effect of position with canopy cover, with edges having less canopy cover than some interior positions. Boreal sites (black bars) showed no edge effect with respect to percent canopy cover (a). Herbaceous cover (b) show an increase in fragment edges in the tropical sites while boreal and temperate sites show no edge effect. Aboveground live tree biomass showed no edge effect in boreal and temperate sites while tropical sites show a mixed effect with edges having less live biomass than some interior positions and no difference from others (c). Total dead woody debris (d) in closed forest sites have a mixed effect in tropical sites, with less dead wood on edges than some (but not all) interior positions. Dead woody debris showed no edge effect in temperate and boreal sites $(\alpha=0.05$, error bars as S.E, $n=$ from 135 to 257 , significantly different positions shown with lower and upper case letters).
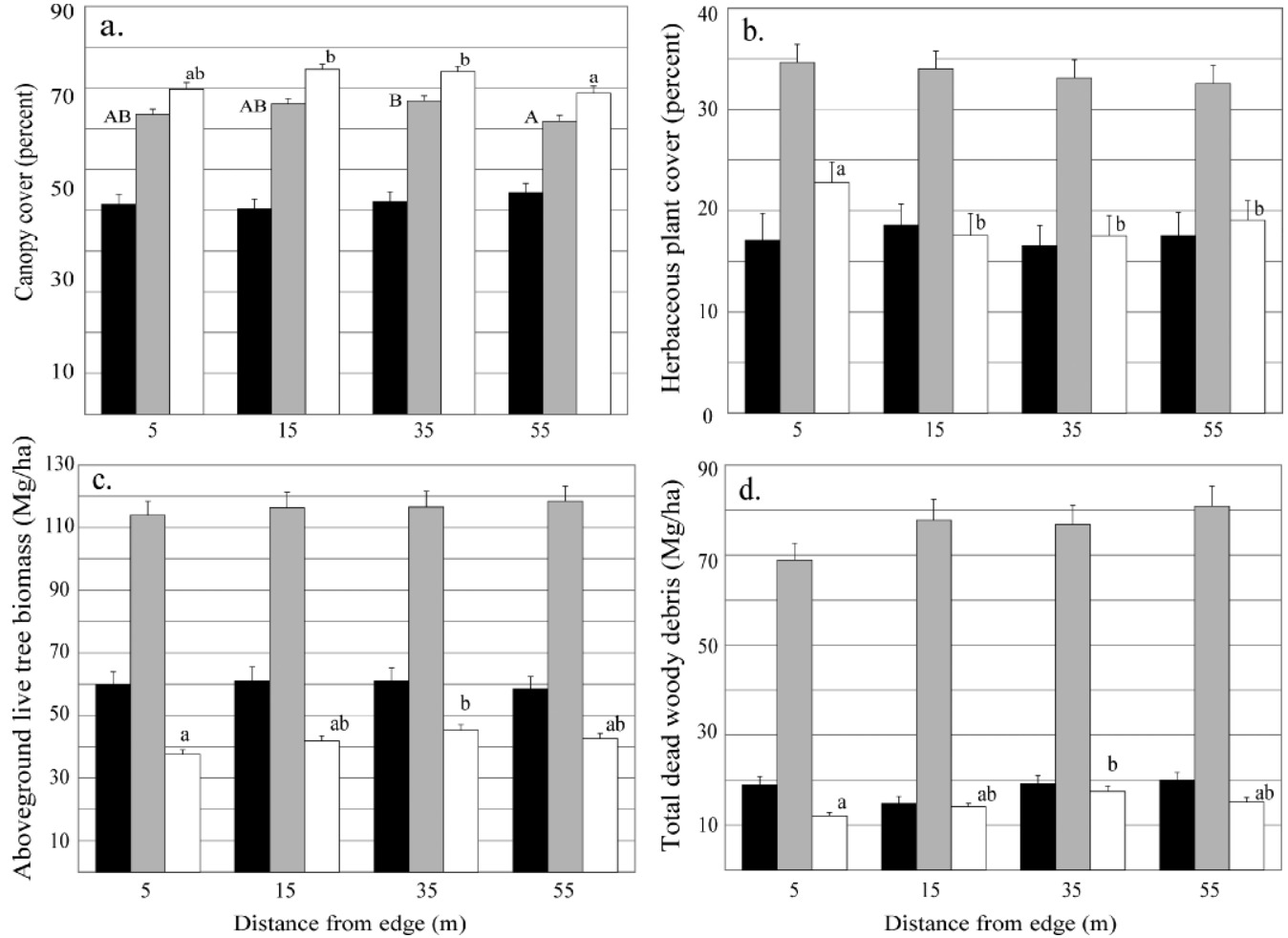


\begin{tabular}{|c|c|c|c|c|c|c|c|c|c|c|c|c|c|c|}
\hline \multicolumn{6}{|c|}{ Temperate } & \multicolumn{9}{|c|}{ Tropical } \\
\hline \multicolumn{3}{|c|}{ Medium } & \multicolumn{3}{|c|}{ Small } & \multicolumn{3}{|c|}{ Large } & \multicolumn{3}{|c|}{ Medium } & \multicolumn{3}{|c|}{ Small } \\
\hline & $\begin{array}{l}\text { Edge } \\
\text { effec }\end{array}$ & & & $\begin{array}{l}\text { Edge } \\
\text { effect }\end{array}$ & & & $\begin{array}{l}\text { Edge } \\
\text { effect }\end{array}$ & & & $\begin{array}{l}\text { Edge } \\
\text { effec }\end{array}$ & & & $\begin{array}{l}\text { Edge } \\
\text { effect }\end{array}$ & \\
\hline $73.8^{\mathrm{a}}$ & 22.3 & - & $74.4^{\mathrm{a}}$ & 20.3 & $\mathrm{x}$ & $80.8^{\mathrm{a}}$ & 24.5 & - & $84.8^{\mathrm{b}}$ & 22.1 & - & $79.5^{\mathrm{a}}$ & 23.2 & - \\
\hline $22.1^{\mathrm{a}}$ & 7.9 & - & $22.6^{\mathrm{a}}$ & 7.3 & - & $7.4^{\mathrm{a}}$ & 2.7 & - & $7.5^{\mathrm{a}}$ & 2.8 & + & $7.2^{\mathrm{a}}$ & 2.4 & - \\
\hline $24.9^{b}$ & 16.1 & - & $28.9^{\mathrm{a}}$ & 16.9 & - & $10.3^{\mathrm{a}}$ & 5.8 & - & $10.6^{\mathrm{a}}$ & 6.2 & - & $10.4^{a}$ & 5.6 & - \\
\hline $2338.7^{\mathrm{b}}$ & 1845.0 & - & $2733.5^{\mathrm{a}}$ & 2150.4 & - & $2829.9^{\mathrm{a}}$ & $3272.2-$ & - & $4030.9^{\mathrm{b}}$ & 7825.8 & - & $3135.2^{\mathrm{a}}$ & 3142.5 & - \\
\hline $14.8^{\mathrm{a}}$ & 5.2 & - & $15.3^{\mathrm{a}}$ & 6.2 & - & $15.4^{\mathrm{a}}$ & 10.2 & - & $15.8^{\mathrm{a}}$ & 12.4 & - & $14.3^{\mathrm{a}}$ & 9.5 & - \\
\hline $1.4^{\mathrm{a}}$ & 1.3 & - & $1.5^{\mathrm{a}}$ & 1.2 & - & $0.7^{\mathrm{a}}$ & 0.5 & - & $0.7^{\mathrm{a}}$ & 0.5 & - & $0.8^{\mathrm{a}}$ & 0.6 & \\
\hline $5.2^{\mathrm{ab}}$ & 5.1 & - & $6.4^{\mathrm{b}}$ & 6.2 & - & $2.4^{\mathrm{a}}$ & 2.4 & - & $2.9^{\mathrm{a}}$ & 3.1 & - & $2.6^{\mathrm{a}}$ & 2.4 & - \\
\hline $9.2^{\mathrm{a}}$ & 9.4 & - & $11.3^{\mathrm{a}}$ & 18.8 & - & $7.2^{\mathrm{a}}$ & 15.2 & - & $5.9^{\mathrm{ab}}$ & 7.6 & - & $5.1^{\mathrm{b}}$ & 6.5 & - \\
\hline $15.8^{\mathrm{b}}$ & 12.6 & - & $19.2^{\mathrm{a}}$ & 21.9 & - & $10.3^{\mathrm{a}}$ & 16.1 & - & $9.5^{\mathrm{a}}$ & 9.1 & - & $8.5^{\mathrm{a}}$ & 7.1 & \\
\hline $29.2^{\mathrm{b}}$ & 34.9 & - & $28.9^{\mathrm{b}}$ & 36.5 & - & $3.1^{\mathrm{a}}$ & 6.6 & - & $2.6^{\mathrm{a}}$ & 6.9 & - & $2.4^{\mathrm{a}}$ & 5.5 & \\
\hline $45.1^{\mathrm{ab}}$ & 40.1 & - & $48.1^{\mathrm{b}}$ & 48.1 & - & $13.4^{\mathrm{a}}$ & 18.2 & - & $12.0^{\mathrm{a}}$ & 12.4 & - & $10.9^{\mathrm{a}}$ & 9.7 & - \\
\hline $14.5^{\mathrm{b}}$ & 28.0 & - & $15.9^{\mathrm{b}}$ & 30.8 & - & $3.0^{\mathrm{a}}$ & 7.3 & - & $1.6^{\mathrm{b}}$ & 6.0 & - & $2.2^{\mathrm{ab}}$ & 5.6 & \\
\hline $59.6^{\mathrm{b}}$ & 54.6 & - & $64.1^{\mathrm{b}}$ & 65.4 & - & $16.4^{\mathrm{a}}$ & 21.0 & - & $13.6^{\mathrm{b}}$ & 14.9 & - & $13.1^{\mathrm{b}}$ & 11.2 & - \\
\hline $3.2^{\mathrm{b}}$ & 2.1 & - & $3.1^{\mathrm{b}}$ & 2.1 & - & 4.3 & 2.4 & & 4.5 & 2.7 & & 4.5 & 2.9 & \\
\hline $7.6^{\mathrm{b}}$ & 7.9 & - & $9.7^{\mathrm{a}}$ & 9.9 & - & 4.8 & 5.1 & - & 4.8 & 6.3 & - & 5.2 & 5.9 & - \\
\hline $10.9^{\mathrm{b}}$ & 8.9 & - & $12.8^{\mathrm{a}}$ & 10.6 & - & 9.1 & 6.6 & - & 9.3 & 7.8 & - & 9.7 & 7.8 & - \\
\hline $105.5^{\mathrm{b}}$ & 71.4 & - & $123.3^{a}$ & 81.6 & - & $42.9^{a}$ & 26.3 & - & $42.7^{\mathrm{a}}$ & 26.2 & + & $39.9^{a}$ & 24.4 & - \\
\hline $21.9^{\mathrm{a}}$ & 22.2 & - & $24.8^{\mathrm{a}}$ & 24.3 & - & $16.5^{\mathrm{a}}$ & 18.2 & - & $15.4^{\mathrm{a}}$ & 20.4 & - & $16.7^{\mathrm{a}}$ & 19.5 & - \\
\hline $37.5^{\mathrm{a}}$ & 30.5 & - & $28.9^{\mathrm{a}}$ & 24.6 & - & $21.0^{\mathrm{a}}$ & 23.9 & - & $20.8^{\mathrm{a}}$ & 24.9 & - & $23.0^{\mathrm{a}}$ & 25.1 & - \\
\hline
\end{tabular}

characteristics in forest stands of differing moisture, i.e., dry and moist forests, structure, i.e., open (typically younger) $v s$. closed (typically older) canopy stands, and size categories, i.e., small (10-14 ha), medium (33 to 60 ha), and large (100-240 ha) along a climatic gradient of boreal, temperate, and tropical sites.

The landscapes we sampled have diverse variables controlling forest structure and biomass, as well as controls that vary nonlinearly across the gradients sampled. For example, the control of topography and aspect as it affects microclimate had a much stronger role in the temperate sites (mountainous) than the boreal sites (non mountainous) or the tropical sites. However, aspect is also an important control on forest structure in Puerto Rico but it is related more to wind and hurricane disturbance than to microclimate. The structure and function of boreal sites in Alaska are strongly influenced by permafrost conditions that do not exist at other sites in our study. Additionally, management varied widely across our sites. Temperate and boreal moist sites were highly managed for timber and fuels while the boreal dry and tropical sites were for the most part unmanaged. Finally, the woody debris and fuels characteristics we sampled are typically very spatially variable components of forest landscapes at fine scales. Nevertheless we attempted to sample enough sites with similar protocol to determine significant trends in forest woody biomass and fuels related to fragmentation under different scenarios of climate, moisture, and stand structure/age.

\section{Temperature}

Woodall and Liknes (24), in an analysis of U.S. Department of Agriculture Forest Service Forest Inventory and Analysis (FIA) data, found a correlation of both CWD and FWD with latitude across the continental United States $\left(20-50^{\circ} \mathrm{N}\right.$. Lat.), with CWD increasing with latitude and FWD showing a similar but weaker relationship. We observed a nonlinear relationship between CWD and FWD with temperature (primarily governed by latitude - but with elevational controls as well). These carbon stocks were greatest in temperate regions and less in both tropical and boreal regions. Likely explanations are both abiotic and biotic as temperature is a predictor of productivity and both temperature and organisms have strong controls on decomposition rates (25). We found greater mean values of CWD and FWD (15.2 and $12.3 \mathrm{Mg} \mathrm{ha}^{-1}$ respectively) vs. those of Woodall and Liknes (24) - calculated as 6.6 and 3.3 for CWD and FWD respectively. This may reflect the differences in the degree of forest closure represented by FIA data (as low as $10 \%$ tree cover) and this data set (mean canopy cover $72.5 \pm 26 \%$ ). However, carbon pool estimates we report for sites in Puerto Rico closely match FIA estimates for Puerto Rico and the USVI (26). We found a strong linear relationship of forest floor fuels with temperature. The duff layers in the boreal forest are a significant component of the fuel load and a very minimal component in the tropical sites - a function of higher decomposition rates of litter in tropical sites related to higher temperatures and organisms (25). A priori we expected to see greater aboveground biomass in the tropical sites relative to temperate and boreal sites in this study but we saw a nonlinear relationship, with the highest values in the temperate sites. Our sites in the tropics reflect three characteristics that are important. They i) occur in an area of regular hurricanes, which strongly affect forest structure by reducing canopy cover and aboveground live biomass for some years after a hurricane (27), ii) are in an area of primarily disturbed forests and secondary growth (28), and iii) include tropical dry forests, typically with lower canopy heights and aboveground biomass than more humid tropical forests (29).

Woodall and Liknes, in a further analysis of FIA data (30), assessed climatic regions as indicators of carbon stocks in the continental US and suggest that this type of analysis may be useful in assessing carbon pools at the continental scale. We also assessed the structural and fuels data by comparing mean values among boreal, temperate, and tropical climatic regions and found significant differences in the means among many of the fuels characteristics. The results show similar trends to the regression analysis with temperature and lend support to the idea that this is a useful approach to quantifying continental scale variation in woody biomass. 


\section{Moisture}

A number of studies report significant differences in forest structure related to moisture $(17,31,32)$. Brandeis and Woodall (26) quantify differences in forest fuels by life zone in Puerto Rico and the USVI and report greater but not significant differences between moist and dry life zones. We found a similar pattern for DWD across all biomes and report significant differences in all fuel categories for the temperate and tropical sites as well as for most of the boreal fuel categories. In nearly all cases fuel loads were greater in moist as opposed to dry sites. The exception is in the duff biomass in the boreal sites, where duff accumulates in the cold dry sites in Alaska, likely due to low rates of decomposition of forest floor carbon pools. The greater amounts of DWD in moist sites may be a reflection of the rate of productivity increasing relative to the rates of decomposition along this gradient. González et al. (14) show increased rates of wood decomposition in tropical dry sites relative to moist sites.

\section{Structure}

We selected older, closed canopy stands and younger, more open canopy stands to test the idea that the edge effects would be greater in the closed stands. We hypothesized that the edge environment would differ more from the interior environment in a mature forest than a young forest. Additionally, fragmented landscapes are often a mosaic of older forest remnants and younger successional stands and we can compare the distribution of woody biomass between these groups along our climatic gradient. Krankina and Harmon (33) report greater stores of dead wood in old growth vs. younger stands in Russian boreal forests. Feller (34) and Hély (35) describe trends in CWD quantity with forest age that suggest a U-shaped curve, with greater quantities occurring in recent cutovers than in oldgrowth forests, and lowest quantities occurring in middle-aged forests. Feller (34) also suggests this may be the normal trend in CWD with forest age, with departures from this trend resulting from disturbance. Other studies have found no relationship between stand age and DWD (36). We saw a pattern of increased live biomass, forest floor fuels, and dead woody debris in the boreal and temperate forests on closed canopy/older stands relative to open canopy/younger stands. This may represent the portion of the curve from middle aged to older forests. We did not see many differences between the open canopy and closed canopy stands in the tropical sites. These forests were similar structurally and may have represented less of a successional gradient than the temperate and boreal sites. Surprisingly our open canopy stands were not significantly different in terms of live tree biomass in boreal temperate and tropical sites, while the closed canopy sites had much greater differences.

\section{Fragment Size}

We selected a range of fragment sizes to test the idea that edge effect would be greater in large fragments, where edge environments could potentially have greater differences from interior environments. The smallest fragments may be virtually all edge and show no difference between edge and interior. Additionally the differing fragment sizes represent typical components of a fragmented landscape. We hypothesized that there may be differences in fuel loads related to fragment size. Laurance et al. (4) suggest that as fragment size decreases the relative amount of edge increases and characteristics of edges i.e. wind throw, tree mortality, species invasions, changes in abiotic conditions and decomposition become relatively more important. This would suggest effects of fragment size would show up in measures of fuels and forest structure attributes. We found greater amounts of total dead woody debris in large fragments vs. medium and small fragments which support this idea. We also found greater live tree biomass in small fragments in boreal and temperate sites. One explanation is that in managed landscapes, small fragments of old forest (with relatively high live tree biomass) may be maintained.

\section{Edge Effects}

Forest edges have been associated with gradients in a number of abiotic and biotic components (4, 37, 38). Conflicting studies have reported greater amounts of DWD (39) and lesser amounts of DWD (40) associated with forest edges. The lack of significant edge effects on DWD in the majority of our fragment types was surprising but may be due in part to the variability in fuels distribution. One of the significant edge effects measured was higher degree of herbaceous cover, typically grasses, in the forest edges relative to the interiors. This herbaceous cover represents a fast drying, fast burning fuel that can serve to bring fire into forest edges where it has the potential to ignite woody fuels.

\section{Conclusion}

Fragmentation of landscapes creates edges. Edges represent the interface of heterogeneous landscape elements and are often very complex environments where organisms, energy, nutrients, and disturbances flow from one component to another. Fire is one such disturbance that often initiates on roadsides and grasslands and burns into forested areas. The behavior of fire at this interface is dependant in part on the carbon stocks and their distribution in forest edges and fragment interiors. In this study, we addressed the question of how fuels are distributed in different fragment types along a climatic gradient, whether there were edge effects related to fuels, and how they vary among fragment types. We found that $i$ ) relationships with climate and carbon stocks are nonlinear, a result of the balance of productivity and decomposition, regulated by temperature and influence by organisms, ii) moisture has strong controls on the amount of fuels in forest fragments, with higher fuel loads in moist versus dry temperate and tropical sites but the opposite relationship in boreal sites with high duff loads, iii) there was less variation among open/younger forest stands among boreal, temperate, and tropical sites than among closed/ older stands, iv) fragment size and edge effects on fuels are present but the variability inherent in fragmented landscapes makes generalizations difficult and effects vary with forest type.

\section{References and Notes}

1. Forman, R.T.T. 1995. Land Mosaics: The Ecology of Landscapes and Regions. Cambridge University Press, Cambridge, UK. 632 pp

Cochrane, M.A. and Laurance, W.F. 2008. Synergisms among fire land use and climate change in the Amazon. Ambio 37, 522-527.

3. Saunders, D.A., Hobbs, R.J. and Margules, C.R. 1991. Biological consequences of ecosystem fragmentation: a review. Conserv. Biol. 5, 18-32.

4. Laurance, W.F., Lovejoy, T.E., Vasconcelos, H.L., Bruna, E.M., Didham, R.K., Stouffer, P.C., Gascon, C., Bierregaard, R.O., et al. 2002. Ecosystem decay of Amazonian forest fragments: a 22-year investigation. Conserv. Biol. 16, 605-618.

. Riitters, K.H., Wickham, J.D., O'Neil, R.V., Jones, K.B., Smith, E.R., Coulston, J.W., Wade, T.G. and Smith, J.H. 2002. Fragmentation of continental United States forests. Ecosystems 5, 815-822.

6. Ranta, P., Blom, T., Niemelä, J., Joensuu, E. and Siitonen, M. 1998. The fragmented Atlantic forest of Brazil: size, shape and distribution of forest fragments. Biodiversity and Atlantic forest of B
Cons. 7, 385-403.

7. Kellman, M., Tackaberry, R. and Rigg, L. 1998. Structure and function in two tropical gallery forest communities: implications for forest conservation in fragmented systems. J. Appl. Ecol. 35, 195-206.

8. Bierregaard, R.O. and Lovejoy, T.E. 1992. The biological dynamics of tropical rainforest fragments. Bioscience. 42, 859-866.

9. Didham, R.K. and Lawton, J.H. 1999. Edge structure determines the magnitude of changes in microclimate and vegetation structure in tropical forest fragments. Biotropica $31,17-30$.

10. Wessman, C.A. and Asner, G.P. 1998. Ecosystems and the problems of large-scale measurements. In: Successes Limitations and Frontiers in Ecosystem Ecology. Groffman, measurements. In: Successes Limitations and Frontiers in Ecosystem Ecology
P. and Pace, M. (eds). Springer-Verlag, Berlin, Germany, pp. 346-371.

11. Weir, J.M., Johnson, E.A. and Miyanishi, K. 2000. Fire frequency and the spatial age mosaic of the mixed-wood boreal forest in western Canada. Ecol. Appl. 10, 1162-1177. 
12. Holdsworth, A.R. and Uhl, C. 1997. Fire in Amazonian selectively logged rainforest and the potential fort fire reduction. Ecol. Appl. 7, 713-725.

13. Cochrane, M.A. and Schultz, M.D. 1998. Fire as a recurrent event in tropical forests of the Eastern Amazon: effects on forest structure, biomass, and species composition. Biotropia 31, 2-16.

14. González, G, Gould, W.A., Hudak, A.T. and Hollingsworth, T.N. 2008. Decay of aspen (Populus tremuloides Michx.) wood in boreal, temperate and tropical forest fragments. Ambio 37, 588-597.

15. Daly, C. and Johnson, G.L. 1999. PRISM spatial climate layers: their development and use. Short course on topics in applied climatology. In: 79th Annual Meeting of the American Meteorological Society. American Meteorological Society, Dallas, TX (http:/ www.ocs.orst.edu/prism/prisguid.pdf)

16. Daly C. 2006. Guidelines for assessing the suitability of spatial climate data sets. Int. J. Climatol. 26, 707-721

17. Ewel, J.J. and Whitmore, J.L. 1973. The Ecological Life Zones of Puerto Rico and the US Virgin Islands. USDA Forest Service Institute of Tropical Forestry, Río Piedras, Puerto Rico, $72 \mathrm{pp}$

18. Gould, W.A., González, G. and Carrero-Rivera, G. 2006. Structure and composition of vegetation along an elevational gradient in Puerto Rico. J. Veg. Sci. 17, 653-664.

19. Meddens, A.J.H., Hudak, A.T., Evans, J.S., Gould, W.A. and González, G. 2008 Characterizing forest fragments in boreal, temperate, and tropical ecosystems. Ambio 37. $569-576$.

20. Harmon, M.E. and Sexton, J. 1996. Guidelines for Measurement of Woody Detritus in Forest Ecosystems. Publication No. 20. USLTER Network Office, Seattle, WA.

21. Scatena, F.N., Silver, W.L., Siccama, T., Johnson, A. and Sanchez, M.J. 1993. Biomass and nutrient content of the Bisley Experimental Watershed, Luquillo Experimenta Forest, Puerto Rico, before and after hurricane Hugo, 1989. Biotropica 25, 15-27.

22. Brown, J.K. 1974. Handbook for Inventorying Downed Woody Material. GTR INT-16. U.S. Department of Agriculture, Forest Service, Intermountain Forest and Range Experiment Station, Ogden, UT, $34 \mathrm{pp}$.

23. SPSS. 2007. SPSS 16.0 for Windows. SPSS Chicago, IL.

24. Woodall, C.W and Liknes, G.C. 2008. Relationships between forest fine and coarse Woody debris carbon stocks across latitudinal gradients in the United States as an indicator of climate change effects. Ecol. Indicators 8, 686-690.

25. González, G. and Seastedt, T.R. 2001. Soil fauna and plant litter decomposition in tropical and subalpine forests. Ecology 82, 955-962

26. Brandeis, T.J. and Woodall, C. W. 2008. Assessment of forest fuel loadings in Puerto Rico and the U.S. Virgin Islands. Ambio 37, 557-562.

27. F.N. Scatena, F.N., Moya, S., Estrada, C. and Chinea, J.D. 1996. The first five years in the reorganization of aboveground biomass and nutrient use following hurricane Hugo in the Bisley Experimental Watershed, Luquillo Experimental Forest, Puerto Rico. Biotropica, 28, 424-440.

28. Brandeis, T.J., Helmer, E.H. and Oswalt, S.N. 2007. The Status of Puerto Rico's Forests, 2003. USDA Forest Service Southern Research Station, Asheville, NC, 75 pp.
29. Brown, S., Lugo, A.E. and Iverson, L.R. 1992. Processes and lands for sequestering carbon in the tropical forest landscape. Water Air Soil Poll. 64, 139-155.

30. Woodall, C.W. and Liknes, G.C. 2008. Climatic regions as an indicator of forest coarse and fine woody debris carbon stocks in the United States. Carbon Balance and Management 3, 5. (http://www.cbmjournal.com/content/3/1/5)

31. Barbour, M.G. and Billings, W.D. 2000. North American Terrestrial Vegetation. Cambridge University Press, 708 pages.

32. Lugo, A. E. 2005. Los bosques. In: Biodiversidad de Puerto Rico-Vertebrados Terrestres y Ecosistemas. Joglar, R.L. (ed). Editorial del Instituto de Cultura Puertoriquenia, San Juan, PR, pp. 397-548

33. Krankina, O.N. and Harmon, M.E. 1995. Dynamics of the dead wood carbon pool in northwestern Russian boreal forests. Water Air Soil Poll. 82, 227-238.

34. Feller, M.C. 2003. Coarse woody debris in the old-growth forests of British Columbia. Environ. Rev. 11, S135-S157.

35. Hély, C., Bergeron, Y. and Flannigan, M.D. 2000. Coarse woody debris in the southeastern Canadian boreal forest: composition and load variations in relation to stand replacement. Can. J. For. Res. 30, 674-687.

36. Ter-Mikaelian, M.T, Colombo, S.J. and Chen, J. 2008. Amount of downed woody debris and its prediction using stand characteristics in boreal and mixedwood forests of Ontario, Canada Can J. For Res. 38, 2189-2197.

37. Chen, J., Franklin, J.F. and Spies, T.A. 1995. Growing-season microclimatic gradients from clearcut edges into old-growth Douglas-fir forests. Ecol. Appl. 5, 74-86.

38. Renhorn, K., Esseen, P., Palmqvuist, K. and Sundberg, B. 1997. Growth and vitality of epiphytic lichens I. Responses to microclimate along a forest edge-interior gradient. Oecologia 109, 1-9.

39. Snäll, T. and Gunnar Jonsson, B. 2001. Edge effects on six polyporous fungi used as oldgrowth indicators in Swedish boreal forest. Ecol. Bull. 49, 255-262.

40. Menzel, M.A., Ford, W.M., Laerm, J. and Krishon, D. 1999. Forest to wildlife opening habitat gradient analysis among small mammals in the southern Appalachians. For. Ecol. Manag. 114, 227-232

41. Acknowledgements. This research was funded by the Joint Fire Science Program project entitled: "Landscape fragmentation and forest fuel accumulation: effects of fragment size, age and climate" (JFSP Project Number 01-1-3-37). The research was also supported by the U.S. Department of Agriculture-Forest Service (USDA-FS), International Institute of Tropical Forestry (IITF). Research at IITF is done in collaboration with UPR. We would like to thank María M. Rivera, Matthew Anderson, Marcos Rodríguez, Ivan Vicens, Adriana Quijano, Juan L. Ramírez, for field work in Puerto Rico. Ben Harlow, Chad Smith, Brenda Nipp, Kasey Prestwich, Curtis Kvamme, and KC Murdock helped with fieldwork in Idaho, Washington, and Minnesota. an Kim Elkin, Ryanne Henigar, Lorelei Walker, and Brian Charlton helped with fieldwork in Alaska. Arjan Meddens helped obtain and organize the database, most importantly the Landsat satellite image and PRISM climate data layers. Maya Quiñones helped with the digital interpretalion of forest fragment sizes. We thank Ariel E. Lugo and Ded with J. Lodge for kindly commenting on an earlier version of this manuscript.
William A. Gould is a landscape ecologist with the USDA Forest Service (FS) International Institute of Tropical Forestry (IITF) and adjunct faculty with the University of Idaho Department of Rangeland Ecology \& Management. He is investigating biodiversity, conservation, land cover and land use patterns and processes in tropical, temperate, and arctic biomes. He leads the Puerto Rico and U.S. Virgin Islands Gap Analysis Programs and the International Institute of Tropical Forestry GIS and Remote Sensing Laboratory. His address: USDA Forest Service, International Institute of Tropical Forestry, 1201 Calle Ceiba, Río Piedras, Puerto Rico 00926-1115, USA

E-mail: wgould@fs.fed.us

Grizelle González is a research ecologist at USDA FS, IITF and adjunct faculty to the Department of Biology, University of Puerto Rico, Río Piedras Campus. Her interests are soil ecology/biology and terrestrial biogeochemistry. Her address: USDA Forest Service, International Institute of Tropical Forestry, 1201 Calle Ceiba, Río Piedras, Puerto Rico 00926-1115, USA.

E-mail: ggonzalez@fs.fed.us

Andrew T. Hudak is a Research Forester specializing in landscapelevel applications of remote sensing for improved ecosystem management at the USDA Forest Service, Rocky Mountain
Research Station, Moscow Forestry Sciences Laboratory. His address: USDA Forest Service, Rocky Mountain Research Station, 1221 South Main Street, Moscow, ID 83843, USA.

E-mail: ahudak@fs.fed.us

Teresa Nettleton Hollingsworth is a research ecologist at USDA FS, Boreal ECOLOGY Cooperative Research Unit, Pacific Northwest Research Station. Her interests are plant community ecology, fire ecology, and vegetation mapping with a focus on boreal ecosystems. Her address: USDA Forest Service, Boreal Forest Cooperative Research Unit. Pacific Northwest Research Station, P.O. Box 756780, University of Alaska Fairbanks 99775, USA

E-mail: thollingsworth@fs.fed.us

Jamie Hollingsworth is the Site Manager for the Bonanza Creek Long Term Ecological Research (LTER) program. He is responsible for two primary research areas, Bonanza Creek Experimental Forest (BCEF) and Caribou/Poker Creeks Research Watershed (CPCRW) including the core LTER data collection. His address: Boreal Ecology Cooperative Research Unit, University of Alaska Fairbanks P.O. Box 756780, Fairbanks, AK 99775, USA.

E-mail: fsjh@uaf.edu 